\title{
Interaction and Communication of Agents in Networks and Language Complexity Estimates
}

\author{
Jan Smid ${ }^{1}$, Marek Obitko ${ }^{2}$, David Fisher ${ }^{3}$, Walt Truszkowski ${ }^{4}$ \\ ${ }^{1}$ Dept. of Computer Sci., Morgan State University, USA, jsmid@jezel morgan. edu \\ ${ }^{2}$ Dept. of Cybernetics, Czech Technical University, obitko@labe.felk.cvut.cz \\ ${ }^{3}$ Software Eng. Inst., Carnegie Mellon University, USA, dfisher@sei.crau.edu \\ ${ }^{4}$ NASA/GSFC 588, Greenbelt, MD 20771, USA, walt.truszkowski@gsfe.nasa.gov
}

\section{Introduction}

Knowledge acquisition and sharing are arguably the most critical activities of communicating agents. We report about our on-going project featuring knowledge acquisition and sharing among communicating agents embedded in a network $[7,8]$. The applications we target range from hardware robots to virtual entities such as internet agents. Agent experiments can be simulated using a convenient simulation language. We analyzed the complexity of communicating agent simulations using Java and Easel [2]. Scenarios we have studied (see also our previous work [6]) are listed below. The communication among agents can range from declarative queries to sub-natural language queries.

- A set of agents monitoring an object are asked to build activity profiles based on exchanging elementary observations.

- A set of car drivers form a line, where every car is following its predecessor. An unsafe distance can create a strong wave in the line. Individual agents are asked to incorporate and apply directions how to avoid the wave.

- A set of micro-air vehicles form a grid and are asked to propagate information and concepts to a central server.

\section{Knowledge Acquisition and Communication}

For given knowledge representation language and agent communication language we follow several principles:

- The agent network is a graph that has short search paths [9], [1].

- The individual agent is a graph that has short search paths.

- Aiil graphs can dynamically change, communities can be formed and communicate.

- The agent understanding substantially depends on the semantic information.

For the knowledge acquisition of agents we use an algorithm that is based on the approach developed by J. Siskind [4]. In short, agents receive a sequence of utterances, each to be paired with a set of conceptual expressions. Conceptual 
expressions are assumed to be provided by e.g. the agent's cognitive system, and consist of conceptual symbols. The basic problem is to map words onto conceptual symbols. The thesis is that the natural language based knowledge representation is effective in representing the agent world.

\section{Simulation Language Complexity}

For knowledge processing as well as for other important agent-related tasks we have studied Easel property-based types (PBT) paradigm [2]. A type is a description of some class of objects, while a description is a set of properties. PBTs are intended to provide a foundation for automated systems that solve problems in ways analogous to those of humans. We further developed our initial comparison of Easel and Java presented in [6]. Java can be extended using special classes, such as Actor that is similar to Easel actor type, which enables lower complexity of programming simulations, such as in Jade [3] agent development environment. However, PBTs are not native structures in Java.

\section{Conclusion}

The presented knowledge acquisition method is promising for the next step of our project that deals with entities equipped with sensors. We have studied several examples of emergent agent systems and described knowledge acquisition and communication and the complexity of the implementation. The complexity of simulation using a specialized language such as Easel is lower compared with a general purpose language such as Java. The drawback of using a new language is the cost of mastering a special purpose language and its syntax rules.

\section{References}

1. A. Barabasi, E. Bonabeau: Scale-Free Networks. Scientific American. May 2003.

2. A. Christie, D. Durkee, D. A. Fisher and D. A. Mundie. Easel Language Reference Manual and Author's Guide. http://www.cert.org/Easel/1rm/index.html

3. F. Bellifemine, A. Poggi, G. Rimassa. JADE - A FIPA-compliant agent framework. Proceedings of PAAM'99. London. 1999

4. J. Siskind. Learning Word-to-meaning Mapping. Models of Language Acquisition. Edited by P. Broeder and J. Murre. Oxford University Press. 2000.

5. J. Smid, M. Obitko, W. Truszkowski. An Approach to Knowledge Exchange and Sharing Between Agents. Innovative Concepts for Agent-Based Systems. Springer Verlag. 2003. LNCS 2534.

6. J. Smid, M. Obitko, V. Snášel. Communicating Agents and Property-Based Types versus Objects. SOFSEM - Current Trends in Computer Science. 2004.

7. J. Smid. Knowledge Models for Network Environment Communications: An Overview. IASTED AIA 2004, PSMP 2 Workshop. Innsbruck. 2004.

8. J. Smid, M.Obitko, V. Snášel. Semantically Based Knowledge Representation. In B.d'Auriol (ed.): The Proceedings of the $\mathrm{CIC}^{\prime} 04$ Conference. Las Vegas. 2004. http://jewel.morgan. edu/ ${ }^{\sim}$ jsmid/psmp3/index.php

9. D. Watts. Small Worlds: The Dynamics of Networks between Order and Randomness. Priceton University Pressq. 1999. 